\section{Pacific Northwest}

National Laboratory

Operated by Battelle for the

U.S. Department of Energy

\title{
Medical Radioisotope Data Survey: 2002 Preliminary Results
}

ER Siciliano

July 2004

Prepared for the U.S. Department of Energy under Contract DE-AC06-76RL01830

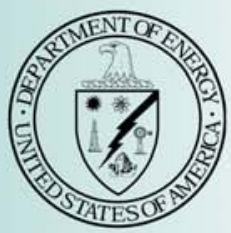




\title{
DISCLAIMER
}

This report was prepared as an account of work sponsored by an agency of the United States Government. Neither the United States Government nor any agency thereof, nor Battelle Memorial Institute, nor any of their employees, makes any warranty, express or implied, or assumes any legal liability or responsibility for the accuracy, completeness, or usefulness of any information, apparatus, product, or process disclosed, or represents that its use would not infringe privately owned rights. Reference herein to any specific commercial product, process, or service by trade name, trademark, manufacturer, or otherwise does not necessarily constitute or imply its endorsement, recommendation, or favoring by the United States Government or any agency thereof, or Battelle Memorial Institute. The views and opinions of authors expressed herein do not necessarily state or reflect those of the United States Government or any agency thereof.

\author{
PACIFIC NORTHWEST NATIONAL LABORATORY \\ operated by \\ BATTELLE \\ for the \\ UNITED STATES DEPARTMENT OF ENERGY
}

under Contract DE-AC06-76RL01830

Printed in the United States of America

Available to DOE and DOE contractors from the

Office of Scientific and Technical Information,

P.O. Box 62, Oak Ridge, TN 37831-0062;

ph: (865) 576-8401

fax: (865) 576-5728

email: reports@adonis.osti.gov

\begin{abstract}
Available to the public from the National Technical Information Service, U.S. Department of Commerce, 5285 Port Royal Rd., Springfield, VA 22161 ph: (800) 553-6847 fax: (703) 605-6900

email: orders@ntis.fedworld.gov

online ordering: http://www.ntis.gov/ordering.htm
\end{abstract}

This document was printed on recycled paper.

$(8 / 00)$ 


\title{
Pacific Northwest \\ National Laboratory
}

Operated by Battelle for the

U.S. Department of Energy

\section{Radiation Portal Monitor Project}

\section{Medical Radioisotope Data Survey: 2002 Preliminary Results}

\author{
E.R. Siciliano
}

June 23, 2004

Prepared for

U.S. Customs and Border Protection under

DOE Contract DE-AC06-76RL01830

Interagency Agreement: 1X03-1461

by

Pacific Northwest National Laboratory

P.O. Box 999

Richland, Washington 99352

Document Number

PIET-43741-TM-197

PNNL Document Number

PNNL-14882 


\section{SUMMARY}

A limited, but accurate amount of detailed information about the radioactive isotopes used in the U.S. for medical procedures was collected from a local hospital and from a recent report on the U.S. Radiopharmaceutical Markets. These data included the total number of procedures, the specific types of procedures, the specific radioisotopes used in these procedures, and the dosage administered per procedure. The information from these sources was compiled, assessed, pruned, and then merged into a single, comprehensive and consistent set of results presented in this report. A summary of these results is given in Table 2-1, and displayed as distributions in Figures $1 \mathrm{a}$ and $1 \mathrm{~b}$.

The main findings from this preliminary survey are:

- $\quad$ A total of approximately 14.4 million medical procedures using radioisotopes were performed in the U.S. during 2001.

- $\quad$ Of this total number of procedures during 2001, approximately 14.2 million were diagnostic procedures and 0.2 million were therapeutic procedures.

- $\quad$ Although there were over 45 different commercially-available products used in over 75 different types of procedures, there were only 17 different isotopes used as radioactive agents in these products.

- $\quad$ Of these 17 different isotopes, 12 are customarily administered to outpatients, and at $91.5 \%$, the isotope Tc-99m is by an overwhelming margin the most likely isotope to be administered. 


\section{CONTENTS}

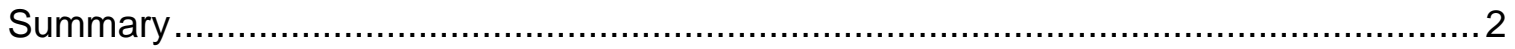

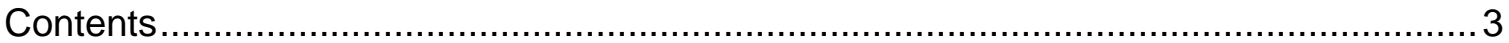

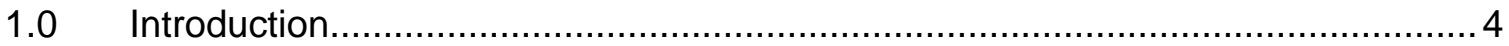

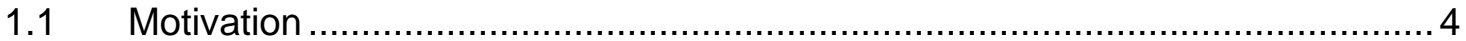

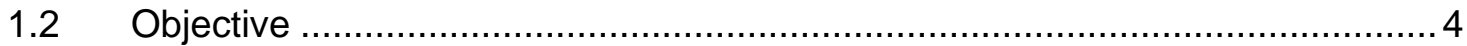

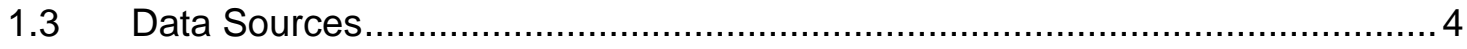

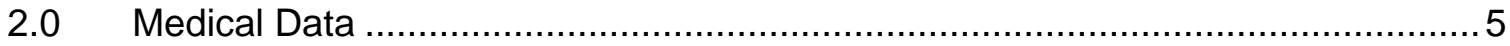

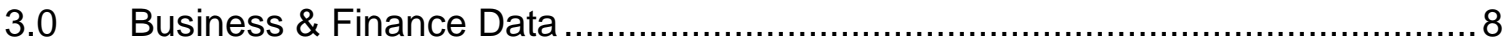

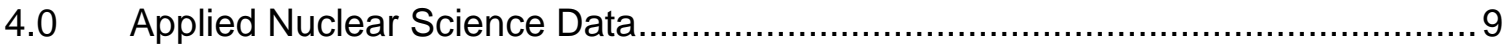

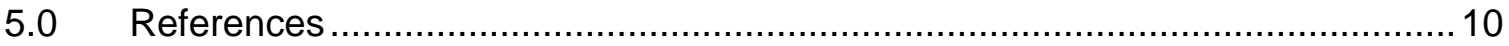

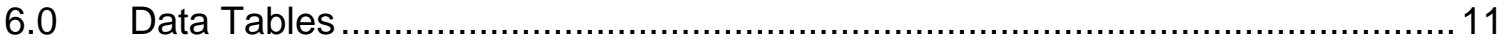

Figure 2-1: FIGURES 1a(Top) and 1b (Bottom). ................................................ 7

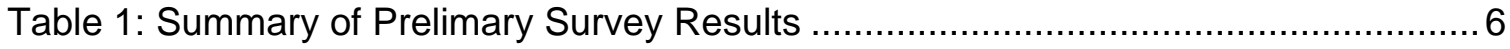

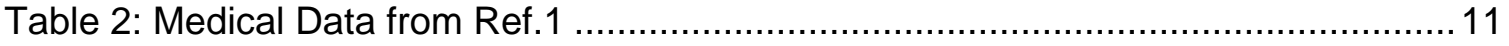

Table 3: Summary of Data from 2001 U.S. Market Survey of Products by Ref.2 ........... 13

Table 4: Medical Isotope Application Data from Ref.3 .......................................... 15 


\subsection{INTRODUCTION}

\subsection{Motivation}

Each day in the U.S., over 39,000 medical procedures are performed that use radioactive isotopes. The overwhelming majority of these procedures are diagnostic in nature, and performed on an out-patient basis, i.e., most of these patients are free to move about in less than 24 hours after receiving the initial dose. Within that time period, most of these patients, traveling either as pedestrians or as passengers in vehicles, may cause alarms in various handheld or roadside radiation detectors. As local governments, as well as the Department of Homeland Security (DHS), Customs and Border Protection (CBP), implements their task to detect the illicit transport of nuclear material, the presence of radiation detectors will increase. The information on medical isotopes compiled in this survey should help in estimating the frequency and signatures of these "innocent" medical-based alarms.

\subsection{Objective}

The objective of this survey was to obtain accurate, up-to-date information about the radioactive isotopes used in the U.S. for medical procedures. The categories of data sought were the total number of such procedures, the specific types of procedures, the specific radioisotopes used in these procedures, and the dosage administered per procedure. The results presented in this Preliminary Survey do not represent an exhaustive search, and thus, should be considered as initial estimates. However, because the data used to obtain these preliminary results was of high quality, the results are expected to be reasonably accurate.

\subsection{Data Sources}

The data collected for this Preliminary Survey was obtained from the three sources (listed in the Reference section, below). These sources represent the Medical community, the Business \& Finance community, and the Applied Nuclear Science community. Because these professions have been compiling information for different purposes, data from all three sources were needed to cover the range of our objectives.

To attain a single, comprehensive and consistent set of data, the information from the above three sources was compiled, assessed, pruned, and then merged to give the results shown in the body of this report. This information is used as an initial database for estimating the number and characteristic signature of medical incidents that may be detected by radiation portal monitors.

A summary of the Preliminary Survey results is given in Table 1, and displayed as distributions in Figure 2-1. The sets of data used to obtain this summary are listed in Table 2, Table 3, and Table 4 in the Data Tables section at the end of this report. Background discussion and more detailed comments about these sets of data are given in the Sections 2, 3, and 4 below. 


\subsection{MEDicAl DATA}

The data from the Medical community were obtained from the records of the Nuclear Medicine Department of a local hospital in Richland WA (Ref.1). The period covered by these records was from January 01, 2001 through November 23, 2002. The original data were listed in two different types of data records: Statistical Report sheets and Exam Master File sheets. The Statistical Report sheets listed the Exam Types, the number of each Exam Type given over the period of the report, and the Patient Type (e.g. In-Patient, Out-Patient, etc.). The Exam Master File sheets listed Exam Reference Numbers (Exam R\#) for each Exam Type, the Radiopharmaceutical Product used for each Exam R\#, and the range of activity for each Radiopharmaceutical Product. The data from these original records were cross-referenced, checked for consistency, and merged into the set of data listed in Table 2 (listed in the Data Tables section at the end of this report).

From the data listed in Table 2, it is seen a total of 2797 exams were performed on 2694 patients (i.e., 1.04 exams per patient) over the base-year period of 1 January $2001-31$ December 2001. The breakdown of these exams was $39.24 \%$ male, $58.20 \%$ female, and $2.41 \%$ pediatric. The majority of these exams (91\%) were administered to out patients. 
Table 1: Summary of Prelimary Survey Results

\begin{tabular}{|c|c|c|c|c|c|c|c|}
\hline Isotope & $\begin{array}{c}\text { Average } \\
\text { Adminis } \\
\text {-tered } \\
\text { Activity } \\
\text { (MBq) }\end{array}$ & Half Life & Organ & $\begin{array}{l}\text { Percent of } \\
2001 \\
\text { Dosages at } \\
\text { local } \\
\text { hospital } \\
\text { (2797 Total) }\end{array}$ & $\begin{array}{l}\text { Percent of } \\
2002 \\
\text { Dosages } \\
\text { at local } \\
\text { hospital } \\
\text { (2352 } \\
\text { Total in } 11 \\
\text { months) }\end{array}$ & $\begin{array}{l}\text { *Percent of } \\
\text { Procedures } \\
\text { Administere } \\
\text { d in U.S. } \\
\text { during } 2001 \\
\text { (14.39 millio } \\
\text { n Total) }\end{array}$ & $\begin{array}{l}\text { *Annual } \\
\text { Procedures in } \\
\text { U.S. per ten } \\
\text { thousand } \\
\text { population for } \\
2001\end{array}$ \\
\hline C-14 & 0.0370 & $5,700 \mathrm{yr}$ & $\begin{array}{l}\text { Kidneys, } \\
\text { Bladder }\end{array}$ & 1.07 & 1.19 & 1.89 & 9.7 \\
\hline Cs-137 & N/A & $30.17 \mathrm{yr}$ & & 0.07 & 0.04 & $\begin{array}{c}\text { Not } \\
\text { Analyzed }\end{array}$ & Not Analyzed \\
\hline Co-57 & 0.0222 & 270 days & $\begin{array}{l}\text { Bladder, } \\
\text { Liver, } \\
\text { Kidneys }\end{array}$ & 0.61 & 1.40 & 1.07 & 5.5 \\
\hline $\mathrm{Cr}-51$ & 2.775 & 27.7 days & Liver & 0.25 & 0.47 & 0.35 & 1.8 \\
\hline F-18 & 740.0 & $109 \mathrm{~min}$ & $\begin{array}{l}\text { Bones, } \\
\text { Bladder }\end{array}$ & *1.43 & *1.00 & 2.02 & 10.3 \\
\hline Ga-67 & 370.0 & $78 \mathrm{hr}$ & $\begin{array}{c}\text { Intestines, } \\
\text { Marrow }\end{array}$ & 0.11 & 0.34 & 0.19 & 1.0 \\
\hline $\mathrm{I}-123$ & 11.10 & $13.3 \mathrm{hrs}$ & $\begin{array}{l}\text { Thyroid, } \\
\text { Kidneys }\end{array}$ & 0.86 & 5.82 & 0.03 & 0.2 \\
\hline I-125 & N/A & 60 day & $\begin{array}{l}\text { Thyroid, } \\
\text { Spleen, } \\
\text { Lungs }\end{array}$ & 0.00 & 0.00 & $\begin{array}{l}\text { Insufficient } \\
\text { Data }\end{array}$ & $\begin{array}{l}\text { Insufficient } \\
\text { Data }\end{array}$ \\
\hline I-131 & 1546.7 & 8 day & $\begin{array}{l}\text { Thyroid, } \\
\text { kidneys }\end{array}$ & 8.54 & 5.19 & 0.34 & 1.7 \\
\hline In-111 & 119.33 & $68 \mathrm{hrs}$ & $\begin{array}{l}\text { Liver, } \\
\text { Marrow, } \\
\text { Spleen }\end{array}$ & 0.89 & 1.49 & 1.40 & 7.2 \\
\hline $\mathrm{Rb}-82 \mathrm{~m}$ & N/A & $6.2 \mathrm{hrs}$ & & 0.00 & 0.00 & $\begin{array}{l}\text { Insufficient } \\
\text { Data }\end{array}$ & $\begin{array}{l}\text { Insufficient } \\
\text { Data }\end{array}$ \\
\hline Sm-153 & 5180.0 & $46.8 \mathrm{hrs}$ & & 0.04 & 0.04 & 0.39 & 2.0 \\
\hline Sr-89 & 148.0 & 50.5 day & & 0.04 & 0.00 & 0.39 & 2.0 \\
\hline Tc-99m & 625.71 & $6 \mathrm{hrs}$ & $\begin{array}{l}\text { Liver, } \\
\text { Spleen, } \\
\text { Marrow, } \\
\text { Kidneys, } \\
\text { Bladder, } \\
\text { Stomach, } \\
\text { Bones }\end{array}$ & 87.06 & 83.55 & 91.51 & 467.7 \\
\hline Tl-201 & 740.00 & $73.5 \mathrm{hrs}$ & $\begin{array}{l}\text { Heart, } \\
\text { Lungs }\end{array}$ & 0.36 & 0.43 & 0.42 & 2.2 \\
\hline Xe-133 & 370.00 & 5 day & Lungs & 0.00 & 0.00 & $\begin{array}{l}\text { Insufficient } \\
\text { Data }\end{array}$ & $\begin{array}{l}\text { Insufficient } \\
\text { Data }\end{array}$ \\
\hline $\mathrm{Y}-90$ & 1184.0 & $64.1 \mathrm{hrs}$ & & 0.00 & 0.04 & $\begin{array}{l}\text { Insufficient } \\
\text { Data }\end{array}$ & $\begin{array}{l}\text { Insufficient } \\
\text { Data }\end{array}$ \\
\hline Footnotes & & & & *Estimated & ${ }^{\star}$ Estimated & $\begin{array}{c}{ }^{*} \text { From Market } \\
\text { Data }\end{array}$ & $\begin{array}{c}\text { *Using } 2000 \\
\text { Census, } \\
281.42 \text { million }\end{array}$ \\
\hline
\end{tabular}



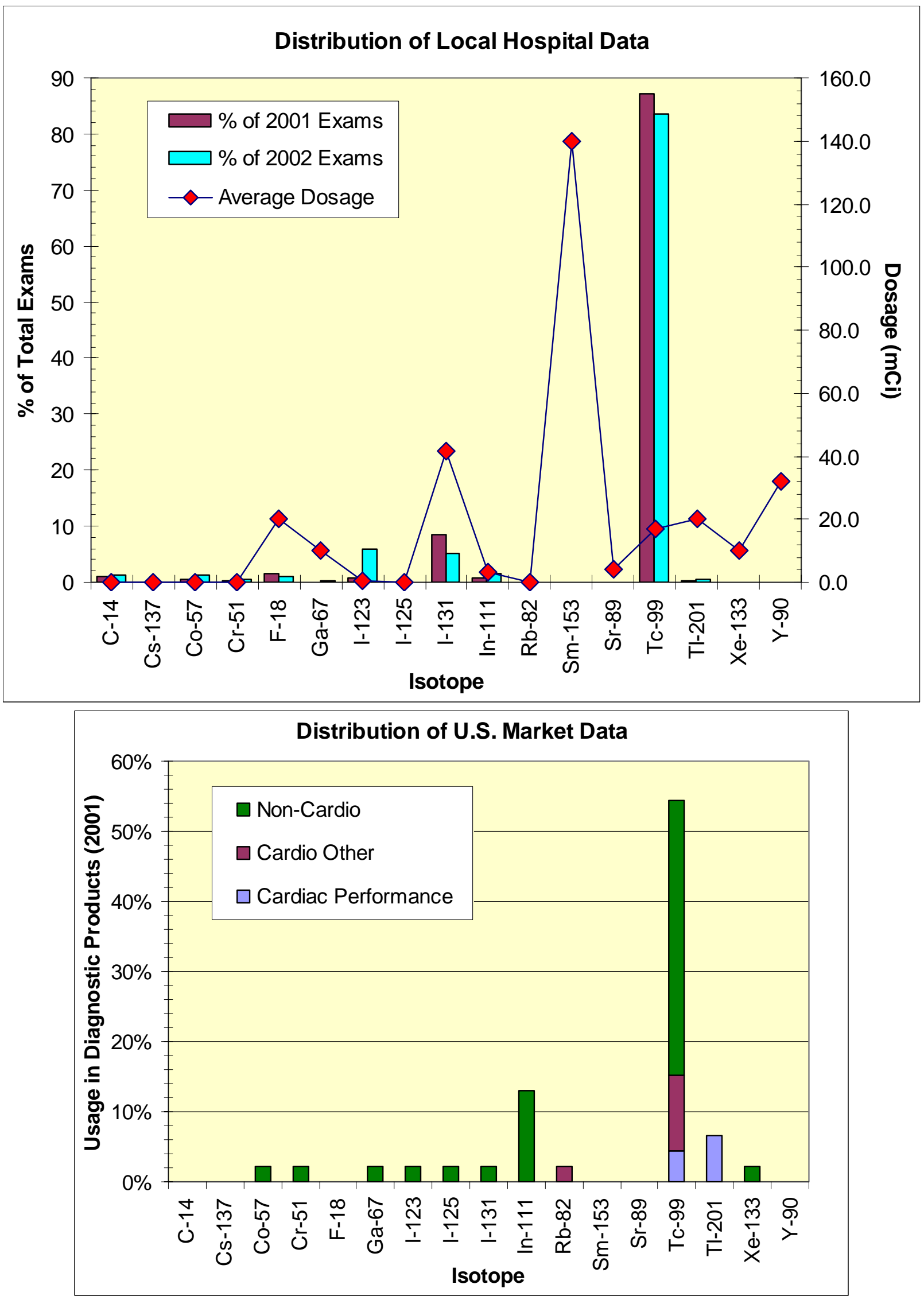

Figure 2-1: FIGURES 1a(Top) and 1b (Bottom). 


\subsection{BUSINESS \& FINANCE DATA}

The data from the Business \& Finance community were taken from a recent report by Frost \& Sullivan entitled "U.S. Radiopharmaceuticals Markets." (Ref.2). The overall objective of this report was to assess the current state of the various sectors of U.S. markets, and predict the behavior of the individual market sectors in the near future. The data in the report covers the eleven-year period from 1998 through 2008; where the 2001 data are used as the base year. The data listed for $2002-2008$ were extrapolated from the earlier data by using reasonably sound economic assumptions, and, depending on the particular market sector, amounted to compound annual growth rates from approximately $2 \%$ to $6 \%$.

Because of the financial perspective of this report, the data from this source is organized according to the dominant market sectors, with emphasis on market-place drivers and projected revenues. Medical procedure types define the market sectors, where the topmost major division of the overall market is made between Diagnostic and Therapeutic procedures. These two major sectors are further broken down into their dominant components of three diagnostic sub- sectors and two therapeutic sub-sectors. These sub-sectors are Diagnostic-Cardiac, Diagnostic-PET/FDG, Diagnostic-Other, and Therapeutic-Bone and Therapeutic-Thyroid. All other therapeutic procedures, such as brachytherapy seeds (Cesium-137 implants) and Rhenium-188 therapy (following vascular stents for prevention of restenosis) were not considered significant for the purposes of this report; and thus were omitted. For each one of the five sub-sectors, we were able to obtain (or deduce) from the information in this report data on the total number of procedures, the dominant products used for these procedures, and the isotope used in each of the products. This information was distributed throughout the report as textual comments or in various tables.

According to this report, there were a total of $14.19 \mathrm{E}+6$ Diagnostic procedures compared to approximately $0.20 \mathrm{E}+6$ Therapeutic procedures performed in the U.S during 2001. The total number of Diagnostic procedures consists of three main categories: 0.29 E+06 FDG (Pet Scans), 5.83 E+06 Cardiology, and 8.08 E+06 Other. The detailed information used to correlate and cross-reference the number of procedures in each of these categories to specific isotopes is summarized in Table 3 (listed in the Data Tables section at the end of this report). 


\subsection{APPLIEd NuClear ScienCe DATA}

The data from the field of Applied Nuclear Science was obtained from R.E. Schenter in the form of a private communication. Dr. Schenter has compiled a comprehensive list of 111 isotopes and their applications from the field of Nuclear Medicine. Note that most of these isotopes have been used in medical research projects, and are not commercially produced. Therefore they are unlikely to be seen at ports of entry into the U.S. Nevertheless, this list is important to have as a reference because some of these isotopes may eventually find their way into the commercial markets. 


\subsection{REFERENCES}

1. VanderMalle, M., Hospital Statistics, Private Communication. 2002.

2. Frost_\&_Sullivan, U.S. Pharmaceutical Markets. 2002.

3. R.E. Schenter, Private Communication 


\subsection{DATA TABLES}

Table 2: Medical Data from Ref.1

\begin{tabular}{|c|c|c|c|c|c|c|}
\hline $\begin{array}{l}\text { Exam } \\
\text { R\# }\end{array}$ & Exam Name & $\begin{array}{l}\text { Radiopharmaceutical } \\
\text { Product }\end{array}$ & Isotope & $\begin{array}{l}\text { Activity } \\
\text { (mCi) }\end{array}$ & $\begin{array}{l}\text { Exams } \\
\text { in } 2001\end{array}$ & $\begin{array}{l}\text { Exams } \\
\text { in } 2002\end{array}$ \\
\hline 1 & ACUTE & Acutect & Tc-99 & 20.0 & 2 & \\
\hline 2 & IN-111 Zevalin & IN-III ZEVALIN & $\ln -111$ & 5.00 & 0 & 1 \\
\hline 3 & Y-90 Zevaline & Y-90 Zevaline & $\mathrm{Y}-90$ & $\begin{array}{l}10.0-- \\
32.0\end{array}$ & 0 & 1 \\
\hline 4 & ADENOSINE Heart Perf. & MYOVIEW & Tc-99 & 22.0 & 204 & 151 \\
\hline 5 & BONE Marrow & Sulfur Colloid & Tc-99 & $\begin{array}{l}10.0-- \\
15.0\end{array}$ & 2 & 4 \\
\hline 6 & BONE SCAN & MDP & Tc-99 & 30.0 & 226 & 184 \\
\hline 7 & BONE SCAN & MDP & Tc-99 & 30.0 & 499 & 416 \\
\hline 8 & BONE SPECT & MDP & Tc-99 & 30.0 & 65 & 49 \\
\hline 9 & BONE Three Phase & MDP & Tc-99 & 30.0 & 58 & 57 \\
\hline 10 & BRAIN Imaging w/ Flow & D.T.P.A. & Tc-99 & 25.0 & 13 & \\
\hline 11 & BRAIN & Ceretec & Tc-99 & 25.0 & 51 & 33 \\
\hline 12 & BREAST Lymphoscintigr & Sulfur Colloid & Tc-99 & $\begin{array}{l}0.90-- \\
1.50\end{array}$ & 20 & 21 \\
\hline 13 & CESIUM 137 Implant & CESIUM 137 Seeds & Cs-137 & ??? & 2 & 1 \\
\hline 14 & CISTERNOGRAPHY & DTPA & In-111 & 1.00 & 3 & 4 \\
\hline 15 & DICOPAC & Co-58/-57 & & 0.0006 & 10 & \\
\hline 16 & DMSA & D.M.S.A & Tc-99 & 4.00 & 0 & 2 \\
\hline 17 & DOBUTAMINE Heart Perf. & Cardiolite & Tc-99 & 22.0 & 14 & 4 \\
\hline 18 & G.E. Reflux & Sulfur Colloid & Tc-99 & 0.50 & 9 & 4 \\
\hline 19 & Gastric Emptying & Sulfur Colloid & Tc-99 & 1.00 & 44 & 57 \\
\hline 20 & GATED & Tc99m- & Tc-99 & $\begin{array}{l}20.0-- \\
30.0\end{array}$ & 39 & 13 \\
\hline 21 & GI Blood Loss & U1traTag RBC & Tc-99 & 20.0 & 19 & 19 \\
\hline 22 & HEPATOBiliary & Hepatolite & Tc-99 & 5.00 & 20 & 32 \\
\hline 23 & HEPATOBILIARY & Hepatolite & Tc-99 & 5.00 & 187 & 180 \\
\hline 24 & LIVERHEMANgioma & U1traTag RBC & Tc-99 & $\begin{array}{l}20.0-- \\
30.0 \\
\end{array}$ & 1 & 1 \\
\hline \multicolumn{7}{|l|}{$\star \star \star 25$} \\
\hline 26 & LIVER/SPLEEN & Microlite & Tc-99 & 6.00 & 4 & 6 \\
\hline 27 & LUNG & MAA & Tc-99 & 6.00 & 42 & 37 \\
\hline 28 & LUNG VENT./DTPA & D.T.P.A. & Tc-99 & $\begin{array}{l}35.0-- \\
40.0\end{array}$ & 41 & 35 \\
\hline 29 & LUNG VENT./ Xe133 & & Xe-133 & 10.0 & 0 & \\
\hline 30 & LYMPHOSCINTGRAPHY & Microlite & Tc-99 & 1.00 & 5 & 3 \\
\hline 31 & MECKELS & Tc99m & Tc-99 & 10.0 & 1 & 6 \\
\hline 32 & MIBI Rest & Cardiolite & Tc-99 & 22.0 & 2 & 60 \\
\hline 33 & MIBI Stress & Cardiolite & Tc-99 & 22.0 & 0 & 43 \\
\hline 34 & MIBI Stress & Cardiolite & Tc-99 & 22.0 & 1 & 20 \\
\hline 35 & MYOVIEW Persanine & MYOVIEW & Tc-99 & 22.0 & 1 & \\
\hline 36 & MYOVIEW Rest & MYOVIEW & Tc-99 & 22.0 & 422 & 279 \\
\hline 37 & MYOVIEW Stress & MYOVIEW & Tc-99 & 22.0 & 217 & 123 \\
\hline 38 & NEOTECT & NeoTect & Tc-99 & $\begin{array}{l}15.0-- \\
20.0\end{array}$ & 1 & \\
\hline 39 & PARATHYROID & Cardlolite & Tc-99 & 25.0 & 12 & 13 \\
\hline
\end{tabular}




\begin{tabular}{|c|c|c|c|c|c|c|}
\hline $\begin{array}{l}\text { Exam } \\
\text { R\# }\end{array}$ & Exam Name & $\begin{array}{l}\text { Radiopharmaceutical } \\
\text { Product }\end{array}$ & Isotope & $\begin{array}{l}\text { Activity } \\
\text { (mCi) }\end{array}$ & $\begin{array}{l}\text { Exams } \\
\text { in } 2001\end{array}$ & $\begin{array}{l}\text { Exams } \\
\text { in } 2002\end{array}$ \\
\hline 40 & PERITONEAL & Sulfur Colloid & Tc-99 & $\begin{array}{l}3.00-- \\
5.00\end{array}$ & 1 & \\
\hline 41 & PY-TEST C14 UREA & C-14 UREA & C-14 & 0.001 & 30 & 28 \\
\hline 42 & PYP INFARCT & PYP & Tc-99 & 25.0 & 0 & 0 \\
\hline 43 & QUADRAMET & Quadremet & Sm-153 & $\begin{array}{l}36.0-- \\
140.0\end{array}$ & 1 & 1 \\
\hline 44 & RED Cell Mass & Cr-51 & Cr-51 & 0.075 & 7 & 11 \\
\hline 45 & RENAL W/ LASIX & MAG3 & Tc-99 & 6.00 & 20 & 24 \\
\hline 46 & RENOGRAM & MAG3 & Tc-99 & 6.00 & 9 & 4 \\
\hline 47 & RENOGRAM w/ Captopril & MAG3 & Tc-99 & 6.00 & 18 & 14 \\
\hline 48 & SCHILLINGS & Co-57 & Co-57 & 0.0005 & 7 & 33 \\
\hline 49 & SCINTIMAMMOGRAPHY & Cardiolite & Tc-99 & 25.0 & 0 & 1 \\
\hline 50 & SHUNT Patency V/P & D.T.P.A. & Tc-99 & 1.00 & 19 & 6 \\
\hline 51 & SPECT Miscellaneous & Ga-67 & Ga-67 & $? ? ?$ & 0 & 1 \\
\hline 52 & STRONTIUM $89 \mathrm{RX}$ & Metastron & Sr-89 & 4.00 & 1 & \\
\hline 53 & THYROID ABLATION & $\mathrm{I}-131$ & $\mathrm{I}-131$ & 30.0 & 18 & 13 \\
\hline 54 & THYROID CA ABLATION & $\mathrm{I}-131$ & $\mathrm{I}-131$ & $\begin{array}{l}34.0-- \\
150.0\end{array}$ & 2 & 4 \\
\hline 55 & $\begin{array}{l}\text { THYROID } \\
\text { Hyperthyroidism }\end{array}$ & $\mathrm{I}-131$ & $\mathrm{I}-131$ & $\begin{array}{l}5.00-- \\
25.0\end{array}$ & 48 & 15 \\
\hline 56 & THYROID SCAN & Tc99m & Tc-99 & 5.00 & 143 & 63 \\
\hline 57 & THYROID SCAN I-123 & I-123 Caps & $\mathrm{I}-123$ & 0.40 & 13 & 75 \\
\hline 58 & THYROID Uptake & $\mathrm{I}-131$ & $\mathrm{I}-131$ & $\begin{array}{l}0.004-- \\
0.016\end{array}$ & 154 & 67 \\
\hline 59 & THYROID Uptake I-123 & I-123 Caps & $\mathrm{I}-123$ & 0.20 & 11 & 62 \\
\hline 60 & THYROID WB Iodine & $\mathrm{I}-131$ & $\mathrm{I}-131$ & $\begin{array}{l}3-- \\
4.00\end{array}$ & 17 & 23 \\
\hline 61 & TL201 REST & TI-201 & TI-201 & 4.00 & 3 & 7 \\
\hline 62 & TL201 24Hour SPECT & Tl-201 & $\mathrm{Tl}-201$ & ??? & 0 & 0 \\
\hline 63 & TL201 ADENOSINE & Tl-201 & TI-201 & 3.50 & 0 & 0 \\
\hline 64 & TL201 PERSANTINE & TI-201 & $\mathrm{TI}-201$ & 3.50 & 0 & 0 \\
\hline 65 & TL201 Reinjection & Tl-201 & Tl-201 & $\begin{array}{l}1.00-- \\
2.00\end{array}$ & 4 & 2 \\
\hline 66 & TL201 Stress Treadmill & TI-201 & TI-201 & 3.50 & 3 & 1 \\
\hline 67 & TRACER SENT OUT & Tc99m & Tc-99 & 20.00 & 0 & 0 \\
\hline 68 & Tumor Localization -M & Cardiolite & Tc-99 & 25.00 & 0 & 0 \\
\hline 69 & Tumor Localization -G & Ga-67 & Ga-67 & $\begin{array}{l}6.00-- \\
10.0\end{array}$ & 3 & 7 \\
\hline 70 & Tumor Localization -O & In-111-0ctreoScan & $\ln -111$ & 6.00 & 9 & 9 \\
\hline \multicolumn{7}{|l|}{$\star \star \star \star 71$} \\
\hline 72 & Tumor Localization -T & TI-201 & TI-201 & 3.50 & 0 & 0 \\
\hline 73 & $\begin{array}{l}\text { Tumor LOCALZ. CEA- } \\
\text { SCA }\end{array}$ & SEA-SCAN & Tc-99 & 25.00 & 0 & 0 \\
\hline 74 & URETERAL Reflux & Tc99m & Tc-99 & 1.00 & 1 & 1 \\
\hline 75 & WHITE Cell / Indium & In-111 WBC & $\ln -111$ & $\begin{array}{l}0.50-- \\
0.90\end{array}$ & 13 & 21 \\
\hline 76 & WHITE Cell / TC-HMPAO & Ceretec & Tc-99 & $\begin{array}{l}8.00-- \\
25.0\end{array}$ & 2 & 0 \\
\hline$\star \star \star 77$ & PET & "FDG" & F-18 & $\begin{array}{l}8.00-- \\
20.0\end{array}$ & 0 & 120 \\
\hline
\end{tabular}


Table 3: Summary of Data from 2001 U.S. Market Survey of Products by Ref.2

\begin{tabular}{|c|c|c|c|c|c|}
\hline Market & $\begin{array}{l}\text { Product } \\
\text { No. }\end{array}$ & Product Name & & & \\
\hline & & & Isotope & Manufacturer & Application (See Notes) \\
\hline \multirow{11}{*}{$\begin{array}{l}\text { Diagnostic } \\
\text { - Cardiac } \\
\end{array}$} & 1 & Cardiolite & Tc-99 & BMS & Cardio/Diag - MP \\
\hline & 2 & Myoview & Tc-99 & Amer. & Cardio/Diag - MP \\
\hline & 3 & Thallium-201 & TI-201 & BMS & Cardio/Diag - MP \\
\hline & 4 & Thallium-201 & TI-201 & Amer. & Cardio/Diag - MP \\
\hline & 5 & Thallium-201 & Tl-201 & Mall. & Cardio/Diag - MP \\
\hline & 6 & Tc-99 PYP & Tc-99 & Amer. & Cardio/Diag - BP \\
\hline & 7 & Phosphotec & Tc-99 & Brac. & Cardio/Diag - BP \\
\hline & 8 & $\begin{array}{l}\text { Technescan } \\
\text { PYP } \\
\end{array}$ & Tc-99 & Mall. & Cardio/Diag - BP \\
\hline & 9 & CIS-Pyro & Tc-99 & CIS & Cardio/Diag - BP \\
\hline & 10 & Pyrolite & Tc-99 & $\mathrm{CIS}$ & Cardio/Diag - BP \\
\hline & 11 & Cardiogen & $\mathrm{Rb}-82$ & Brac. & Cardio/Diag - MV \\
\hline $\begin{array}{l}\text { Diagnostic } \\
\text { - Other }\end{array}$ & 12 & $\begin{array}{l}\text { Sodium } \\
\text { Chromate }\end{array}$ & Cr-51 & Mall. & $\mathrm{RBC}$ \\
\hline \multirow[t]{20}{*}{$\begin{array}{l}\text { (Tier-1 } \\
\text { Suppliers) }\end{array}$} & 13 & *no name* & Ga-67 & $\begin{array}{l}\text { Amer., BMS, } \\
\text { Mall. }\end{array}$ & $\begin{array}{l}\text { Soft Tissue Tumor / } \\
\text { Lymphoma }\end{array}$ \\
\hline & 14 & Sodium lodine & $\mathrm{I}-123$ & Amer., Mall. & Thyroid \\
\hline & 15 & $\begin{array}{l}\text { Human Serum } \\
\text { Albmn }\end{array}$ & $\mathrm{I}-125$ & Mall. & Blood / Plasma Vol. \\
\hline & 16 & Chloride & $\ln -111$ & Amer., Mall. & Labelling Agent \\
\hline & 17 & Oxine & $\ln -111$ & Amer. & WBC / platelets \\
\hline & 18 & Pentetreotide & $\ln -111$ & Mall. & Neuroendoctrine Tumors \\
\hline & 19 & Bicisate & Tc-99 & BMS & Brain \\
\hline & 20 & Exametazine & TC-99 & Amer. & $\begin{array}{l}\text { Cerebral Blood Flow I } \\
\text { WBC }\end{array}$ \\
\hline & 21 & MAA & Tc-99 & Mall. & Lung \\
\hline & 22 & Medronate & Tc-99 & Amer., Mall. & Skeletal / Bone \\
\hline & 23 & Mertiatide & Tc-99 & Mall. & Renal \\
\hline & 24 & Oxidronate & Tc-99 & Amer., Mall. & Bone \\
\hline & 25 & Pentetate & Tc-99 & Amer., Mall. & Kidney / Brain \\
\hline & 26 & Pyrophosphate & Tc-99 & Amer., Mall. & Skeletal / Cardiac \\
\hline & 27 & RBC & TC-99 & Mall. & Blood Pool / GI \\
\hline & 28 & Sestamibi & Tc-99 & BMS & Cardiac / Breast \\
\hline & 29 & $\begin{array}{l}\text { Sdm. } \\
\text { Glucoheptonat } \\
\text { e }\end{array}$ & Tc-99 & Mall. & Kidney / Brain \\
\hline & 30 & Succimer & Tc-99 & Amer. & Renal Parenchymal \\
\hline & 31 & Sulfur Colloid & Tc-99 & Amer. & $\mathrm{RC}$ \\
\hline & 32 & ${ }^{*}$ no name* & Xe-133 & $\begin{array}{l}\text { Amer., BMS, } \\
\text { Mall. }\end{array}$ & Pulmonary / Lung \\
\hline $\begin{array}{l}\text { Diagnostic } \\
\text { - Other }\end{array}$ & 33 & Cyanobalamin & Co-57 & Brac. & Pernicious Anemia \\
\hline
\end{tabular}




\begin{tabular}{|c|c|c|c|c|c|}
\hline $\begin{array}{l}\text { (Tier-2 } \\
\text { Suppliers) }\end{array}$ & 34 & $\begin{array}{l}\text { Capromab } \\
\text { Pendetide }\end{array}$ & In-111 & Cytg & Prostate Cancer \\
\hline & 35 & $\begin{array}{l}\text { Satumomab } \\
\text { Pendetide }\end{array}$ & $\ln -111$ & Cytg & $\begin{array}{l}\text { Colorectal / Ovarian } \\
\text { Cancer }\end{array}$ \\
\hline & 36 & Pentetreotide & $\ln -111$ & $\mathrm{CIS}$ & CSF Kinetics \\
\hline & 37 & MIBG & $\mathrm{I}-131$ & CIS, Drax. & $\begin{array}{l}\text { Pheochromocytomas / } \\
\text { Neuroblastoma }\end{array}$ \\
\hline & 38 & Apcitide & Tc-99 & Brlx & Acute Venous Thrombosis \\
\hline & 39 & Arcitumomab & Tc-99 & Immu & Colorectal Cancer \\
\hline & 40 & Depreotide & Tc-99 & Brlx & Pulmonary Mass \\
\hline & 41 & Disofenin & Tc-99 & $\mathrm{CIS}$ & Hepatobiliary \\
\hline & 21 & MAA & Tc-99 & Brac., CIS & Lung / Perfusion \\
\hline & 42 & Mebrofenin & Tc-99 & Brac., CIS & Hepatobiliary \\
\hline & 25 & Pentetate & Tc-99 & $\begin{array}{l}\text { Brac., CIS, } \\
\text { Drax. }\end{array}$ & Kidney / Brain \\
\hline & 26 & Pyrophosphate & Tc-99 & Brac., CIS & Skeletal / Cardiac \\
\hline & 29 & $\begin{array}{l}\text { Sodium } \\
\text { Glucoheptonate }\end{array}$ & Tc-99 & Drax. & Kidney / Brain \\
\hline & 31 & Sulfur Colloid & Tc-99 & $\mathrm{CIS}$ & $\mathrm{RC}$ \\
\hline & 32 & *no name* & Xe-133 & Drax. & Lung / Pulmonary \\
\hline $\begin{array}{l}\text { Diagnostic - } \\
\text { PET }\end{array}$ & 43 & FDG & F-18 & & PET Diagnostic \\
\hline $\begin{array}{l}\text { Therapeutic- } \\
\text { Bone }\end{array}$ & 44 & Quadramet & $\begin{array}{l}\text { Sm- } \\
153\end{array}$ & & Bone Pain Palliation \\
\hline $\begin{array}{l}\text { (55-60\% of } \\
\text { Therapy } \\
\text { Sector) } \\
\end{array}$ & 45 & Metastron & Sr-89 & & Bone Pain Palliation \\
\hline $\begin{array}{l}\text { Therapeutic- } \\
\text { Thyroid }\end{array}$ & 46 & *no name* & $\mathrm{I}-131$ & & $\begin{array}{l}\text { Hyperthyroidism/Thyroid } \\
\text { Cancer }\end{array}$ \\
\hline $\begin{array}{l}\text { (40-45\% of } \\
\text { Therapy } \\
\text { Sector) }\end{array}$ & & & & & \\
\hline
\end{tabular}

NOTES:

Products listed Market Sector, then top-down by percent.

Some products are supplied by more than one manufacturer.

Application Key

\begin{tabular}{ll}
\hline$M P=>$ & Myocardial Perfusion \\
$B P=>$ & Blood Pool \\
$M V=>$ & Myocardial Viability \\
RBC $=>$ & Red Blood Cells \\
WBC $=>$ & White Blood Cells \\
GI $=>$ & Gastrointestinal \\
RC $=>$ & Reticuloendothelial Cells \\
$C S F=>$ &
\end{tabular}


Table 4: Medical Isotope Application Data from Ref.3.

\begin{tabular}{|c|c|c|}
\hline ISOTOPE & Half-Life & APPLICATIONS \\
\hline Ac-225 & $10.0 d$ & Monoclonal antibody attachment used for cancer treatment (RIT), also parent of Bi-213. \\
\hline Ac-227 & $21.8 y$ & Parent of Ra-223 (Monoclonal antibody attachment used for cancer treatment (RIT). \\
\hline Am-241 & $432 y$ & Osteoporosis detection, heart imaging. \\
\hline As-72 & 26.0h & Planar imaging, SPECT or PET. \\
\hline As-74 & $17.8 \mathrm{~d}$ & Positron-emitting isotope with biomedical applications. \\
\hline At-211 & $7.21 \mathrm{~h}$ & $\begin{array}{l}\text { Monoclonal antibody attachment (alpha emitter) used for cancer treatment (RIT), used } \\
\text { with F-18 for in vivo studies. }\end{array}$ \\
\hline Au-198 & $2.69 d$ & Cancer treatment using mini-gun (B), treating ovarian, prostate, and brain cancer. \\
\hline B-11 & Stable & Melanoma and brain tumor treatment. \\
\hline $\mathrm{Be}-7$ & $53.2 d$ & Used in berylliosis studies. \\
\hline $\mathrm{Bi}-212$ & $1.10 \mathrm{~h}$ & $\begin{array}{l}\text { Monoclonal antibody attachment (alpha emitter) used for cancer treatment (RIT), } \\
\text { cellular dosimetry studies. }\end{array}$ \\
\hline Bi-213 & $45.6 \mathrm{~m}$ & Monoclonal antibody attachment (alpha emitter) used for cancer treatment (RIT). \\
\hline $\mathrm{Br}-75$ & $98 m$ & Planar imaging, SPECT or PET (C). \\
\hline $\mathrm{Br}-77$ & $57 \mathrm{~h}$ & $\begin{array}{l}\text { Label radiosentizers for Te quantization of hypoxia in tumors, and monoclonal antibody } \\
\text { labeling. }\end{array}$ \\
\hline C-11 & $20.3 m$ & Radiotracer in PET scans to study normal/abnormal brain functions. \\
\hline C-14 & $5730 y$ & Radiolabeling for detection of tumors (breast, et al.). \\
\hline Ca-48 & Stable & \\
\hline Cd-109 & $462 d$ & Cancer detection (C), pediatric imaging (C). \\
\hline Ce-139 & $138 d$ & Calibrates high-purity germanium gamma detectors <medical application?>. \\
\hline Ce-141 & $32.5 d$ & Gastrointestinal tract diagnosis, measuring regional myocardial blood flow. \\
\hline Cf-252 & $2.64 y$ & Cervical, melanoma, brain cancer treatment. \\
\hline Co-55 & $17.5 \mathrm{~h}$ & $\begin{array}{l}\text { Planar imaging, SPECT or PET (B). Used in PET imaging of damaged brain tissue after } \\
\text { stroke. }\end{array}$ \\
\hline Co-57 & $272 d$ & $\begin{array}{l}\text { Gamma camera calibration, should be given high priority, radiotracer in research and a } \\
\text { source for X-ray fluorescence spectroscopy. }\end{array}$ \\
\hline Co-60 & $5.27 y$ & $\begin{array}{l}\text { Teletherapy (destroy cancer cells), disinfect surgical equipment and medicines, } \\
\text { external radiation cancer therapy (E). }\end{array}$ \\
\hline Cr-51 & $27.7 d$ & Medical, cell labeling and dosimetry. \\
\hline Cs-130 & $29.2 \mathrm{~m}$ & Myocardial localizing agent. \\
\hline Cs-131 & $9.69 d$ & Intracavity implants for radiotherapy. \\
\hline Cs-137 & $30.2 y$ & Blood irradiators, PET imaging, tumor treatment. \\
\hline Cu-61 & $3.35 \mathrm{~h}$ & Planar imaging, SPECT or PET (B). \\
\hline Cu-62 & $4.7 \mathrm{~m}$ & $\begin{array}{l}\text { Positron emitting radionuclide (B), cerebral and myocardial blood flow used As-a tracer } \\
\text { in conjunction with } \mathrm{Cu} 64(\mathrm{~B}) \text {. }\end{array}$ \\
\hline Cu-64 & $12.7 \mathrm{~h}$ & $\begin{array}{l}\text { PET scanning }(C) \text {, planar imaging }(C), \text { SPECT imaging }(C) \text { dosimetry studies }(C) \text {, } \\
\text { cerebral and myocardial blood flow }(C) \text {, used with Cu-62 (C), treating of colorectal } \\
\text { cancer. }\end{array}$ \\
\hline Cu-67 & $61.9 \mathrm{~h}$ & $\begin{array}{l}\text { Cancer treatment/diagnostics, monoclonal antibodies, radioimmunotherapy, planar } \\
\text { imaging, SPECT or PET. }\end{array}$ \\
\hline Dy-165 & $2.33 \mathrm{~h}$ & Radiation synovectomy, rheumatoid arthritis treatment. \\
\hline Eu-152 & $13.4 \mathrm{y}$ & Medical. \\
\hline Eu-155 & $4.73 y$ & Osteoporosis detection. \\
\hline
\end{tabular}




\begin{tabular}{|c|c|c|}
\hline ISOTOPE & Half-Life & APPLICATIONS \\
\hline F-18 & $110 \mathrm{~m}$ & Radiotracer for brain studies (C), PET imaging (C). \\
\hline Fe-55 & $2.73 y$ & Heat source <medical application?>. \\
\hline Fe-59 & $44.5 d$ & Medical. \\
\hline Ga-64 & $2.63 \mathrm{~m}$ & Treatment of pulmonary diseases ending in fibrosis of lungs. \\
\hline Ga-67 & $78.3 \mathrm{~h}$ & $\begin{array}{l}\text { Imaging of abdominal infections (C), detect Hodgkins/non-Hodgkins lymphoma (C), } \\
\text { used with In-111 for soft tissue infections and osteomyelitis detection (C), evaluate } \\
\text { sarcoidiodis and other granulomaous diseases, particularly in lungs and mediastiusim } \\
\text { (C). }\end{array}$ \\
\hline Ga-68 & $68.1 \mathrm{~m}$ & $\begin{array}{l}\text { Study thrombosis and atherosclerosis, PET imaging, detection of pancreatic cancer, } \\
\text { attenuation correction. }\end{array}$ \\
\hline Gd-153 & $242 d$ & Dual photon source, osteoporosis detection, SPECT imaging. \\
\hline Ge-68 & 271d & PET imaging. \\
\hline $\mathrm{H}-3$ & $12.3 y$ & Labeling, PET imaging. \\
\hline $\mathrm{I}-122$ & $3.6 \mathrm{~m}$ & Brain blood flow studies. \\
\hline $\mathrm{I}-123$ & $13.1 \mathrm{~h}$ & $\begin{array}{l}\text { Brain, thyroid, kidney, and myocardial imaging (C), cerebral blood flow (ideal for } \\
\text { imaging) (C), neurological disease (Alzheimer's) (C). }\end{array}$ \\
\hline $\mathrm{I}-124$ & $4.17 d$ & Radiotracer used to create images of human thyroid, PET imaging. \\
\hline $\mathrm{I}-125$ & $59.9 d$ & $\begin{array}{l}\text { Osteoporosis detection, diagnostic imaging, tracer for drugs, monoclonal antibodies, } \\
\text { brain cancer treatment (I-131 replacement), SPECT imaging, radiolabeling, tumor } \\
\text { imaging, mapping of receptors in the brain }(A) \text {, interstitial radiation therapy } \\
\text { (brachytherapy) for treatment of prostate cancer }(E) \text {. }\end{array}$ \\
\hline $\mathrm{I}-131$ & 8.04d & $\begin{array}{l}\text { Lymphoid tissue tumor/hyperthyroidism treatment }(C) \text {, antibody labeling }(C) \text {, brain } \\
\text { biochemistry in mental illness }(C) \text {, kidney agent }(C) \text {, thyroid problems }(C) \text {, alternative to } \\
\text { Tl-201 for radioimmunotherapy }(C) \text {, imaging, cellular dosimetry, scintigraphy, treatment } \\
\text { of graves disease, treatment of goiters, SPECT imaging, treatment of prostate cancer, } \\
\text { treatment of hepatocellular carcinoma, treatment of melanoma }(A) \text {, locate osteomyelitis } \\
\text { infections }(A) \text {, radiolabeling }(A) \text {, localize tumors for removal }(A) \text {, treatment of spinal } \\
\text { tumor }(A) \text {, locate metastatic lesions }(A) \text {, treAt-neuroblastoma }(A) \text {, internal (systemic) } \\
\text { radiation therapy }(E) \text {, treatment of carcinoma of the thyroid }(E) \text {. }\end{array}$ \\
\hline $\mathrm{I}-132$ & $2.28 \mathrm{~h}$ & Mapping precise area of brain tumor before operating. \\
\hline In-111 & 2.81d & $\begin{array}{l}\text { Detection of heart transplant rejection (C), imaging of abdominal infections (C), } \\
\text { antibody labeling }(C) \text { cellular immunology (C), used with Ga-67 for soft tissue infection } \\
\text { detection and ostemyelitis detection (C), concentrates in liver, kidneys (C), high specific } \\
\text { activity (C), white blood cell imaging, cellular dosimetry, myocardial scans, treatment of } \\
\text { leukemia, imaging tumors. }\end{array}$ \\
\hline In-115m & $4.49 \mathrm{~h}$ & Label blood elements for evaluating inflammatory bowel disease. \\
\hline Ir-191m & $6 s$ & Cardiovascular angiography. \\
\hline Ir-192 & $73.8 \mathrm{~d}$ & $\begin{array}{l}\text { Implants or "seeds" for treatment of cancers of the prostate, brain, breast, } \\
\text { gynecological cancers. }\end{array}$ \\
\hline $\mathrm{Kr}-81 \mathrm{~m}$ & $13.3 \mathrm{~s}$ & Lung imaging. \\
\hline Lu-177 & $6.68 d$ & Heart disease treatment (restenosis therapy), cancer therapy. \\
\hline Mn-51 & $46.2 \mathrm{~m}$ & Myocardial localizing agent. \\
\hline Mn-52 & $5.59 d$ & PET scanning. \\
\hline Mo-99 & $65.9 \mathrm{~h}$ & Parent for Tc-99m generator used for brain, liver, lungs, heart imaging. \\
\hline $\mathrm{N}-13$ & $9.97 \mathrm{~m}$ & PET imaging, myocardial perfusion. \\
\hline $\mathrm{Nb}-95$ & $35 d$ & $\begin{array}{l}\text { Study effects of radioactivity on pregnant women and fetus, myocardial tracer, PET } \\
\text { imaging. }\end{array}$ \\
\hline O-15 & $122 \mathrm{~s}$ & Water used for tomographic measuring of cerebral blood flow (C), PET imaging (C), \\
\hline
\end{tabular}




\begin{tabular}{|c|c|c|}
\hline ISOTOPE & Half-Life & APPLICATIONS \\
\hline & & SPECT imaging. \\
\hline Os-191 & $15.4 d$ & Parent for Ir-191m generator used for cardiovascular angiography. \\
\hline Os-194 & $6.00 y$ & Monoclonal antibody attachment used for cancer treatment (RIT). \\
\hline P-32 & $14.3 d$ & $\begin{array}{l}\text { Polycythaemia Rubra Vera (blood cell disease) and leukemia treatment, bone disease } \\
\text { diagnosis/treatment, SPECT imaging of tumors }(A) \text {, pancreatic cancer treatment }(A) \text {, } \\
\text { radiolabeling }(A) \text {. }\end{array}$ \\
\hline P-33 & $25 d$ & Labeling. \\
\hline $\mathrm{Pb}-203$ & $2.16 d$ & $\begin{array}{l}\text { Planar imaging, SPECT or PET (used with Bi-212) (B), monoclonal antibody } \\
\text { immunotherapy (B), cellular dosimetry. }\end{array}$ \\
\hline $\mathrm{Pb}-212$ & $10.6 \mathrm{~h}$ & Radioactive label for therapy using antibodies, cellular dosimetry. \\
\hline Pd-103 & $17 d$ & Prostate cancer treatment. \\
\hline Pd-109 & $13.4 \mathrm{~h}$ & Potential radiotherapeutic agent. \\
\hline $\mathrm{Pu}-238$ & $2.3 y$ & Pacemaker (no Pu-236 contaminants). \\
\hline Ra-223 & $11.4 d$ & Monoclonal antibody attachment (alpha emitter) used for cancer treatment (RIT). \\
\hline Ra-226 & $1.60 \mathrm{e} 3 \mathrm{y}$ & $\begin{array}{l}\text { Target isotope to make Ac-227, Th-228, Th-229 (Parents of alpha emitters used for } \\
\text { RIT). }\end{array}$ \\
\hline $\mathrm{Rb}-82$ & $1.27 \mathrm{~m}$ & $\begin{array}{l}\text { Myocardial imaging agent, early detection of coronary artery disease, PET imaging, } \\
\text { blood flow tracers. }\end{array}$ \\
\hline Re-186 & $3.9 d$ & $\begin{array}{l}\text { Cancer treatment/diagnostics, monoclonal antibodies, bone cancer pain relief, } \\
\text { treatment of rheumatoid arthritis, treatment of prostate cancer, treating bone pain. }\end{array}$ \\
\hline Re-188 & $17 \mathrm{~h}$ & Monoclonal antibodies, cancer treatment. \\
\hline Rh-105 & $35.4 \mathrm{~h}$ & $\begin{array}{l}\text { Potential therapeutic applications: target neoplastic cells (e.g., small cell lung cancer) } \\
\text { (A), labeling of molecules and monoclonal antibodies }(A) \text {. }\end{array}$ \\
\hline Ru-97 & $2.89 d$ & $\begin{array}{l}\text { Monoclonal antibodies label (C), planar imaging (C), SPECT or PET techniques (C), } \\
\text { gamma-camera imaging. }\end{array}$ \\
\hline Ru-103 & 39d & Myocardial blood flow, radiolabeling mircospheres, PET imaging. \\
\hline S-35 & $87.2 \mathrm{~d}$ & Nucleic acid labeling, P-32 replacement, cellular dosimetry. \\
\hline Sc-46 & $84 d$ & Regional blood flow studies, PET imaging. \\
\hline Sc-47 & 3.34d & Cancer treatment/diagnostics $(F)$, monoclonal antibodies $(F)$, radioimmunotherapy $(F)$. \\
\hline Se-72 & $8.4 d$ & Brain imaging, generator system with As-72, monoclonal antibody immunotherapy. \\
\hline Se-75 & 120d & Radiotracer used in brain studies, scintigraphy scanning. \\
\hline Si-28 & Stable & Radiation therapy of cancer. \\
\hline Sm-145 & 340d & Brain cancer treatment using I-127 (D). \\
\hline Sm-153 & $2.00 d$ & $\begin{array}{l}\text { Cancer treatment/diagnostics }(C), \text { monoclonal antibodies }(C) \text {, bone cancer pain relief } \\
\text { (C), higher uptake in diseased bone than Re-186 (C), treatment of leukemia. }\end{array}$ \\
\hline Sn-117m & $13.6 \mathrm{~d}$ & Bone cancer pain relief. \\
\hline Sr-85 & $65.0 \mathrm{~d}$ & Detection of focal bone lesions, brain scans. \\
\hline Sr-89 & $50 d$ & $\begin{array}{l}\text { Bone cancer pain palliation (improves the quality of life), cellular dosimetry, treatment of } \\
\text { prostate cancer, treatment of multiple myeloma, osteoblastic therapy, potential agent } \\
\text { for treatment of bone metastases from prostate and breast cancer (E). }\end{array}$ \\
\hline Sr-90 & $29.1 y$ & Generator system with Y-90 (B), monoclonal antibody immunotherapy (B). \\
\hline Ta-178 & $9.3 \mathrm{~m}$ & Radionuclide injected into patients to allow viewing of heart and blood vessels. \\
\hline Ta-179 & $1.8 y$ & $\begin{array}{l}\text { X-ray fluorescence source and in thickness gauging (might be a good substitute for } \\
\text { Am-241). }\end{array}$ \\
\hline Ta-182 & $115 d$ & Bladder cancer treatment, internal implants. \\
\hline Tb-149 & $4.13 \mathrm{~h}$ & Monoclonal antibody attachment used for cancer treatment (RIT). \\
\hline
\end{tabular}




\begin{tabular}{|c|c|c|}
\hline ISOTOPE & Half-Life & APPLICATIONS \\
\hline Tc-96 & $4.3 d$ & Animal studies with Tc-99m. \\
\hline Tc-99m & $6.01 \mathrm{~h}$ & $\begin{array}{l}\text { Brain, heart, liver (gastoenterology), lungs, bones, thyroid, and kidney imaging }(C) \text {, } \\
\text { regional cerebral blood flow (C), equine nuclear imaging (C), antibodies (C), red blood } \\
\text { cells (C), replacement for TI-201 (C). }\end{array}$ \\
\hline Th-228 & $720 d$ & Cancer treatment, monoclonal antibodies, parent of Bi-212. \\
\hline Th-229 & $7300 y$ & $\begin{array}{l}\text { Grandparent for alpha emitter (Bi-213) used for cancer treatment (RIT), parent of Ac- } \\
225 .\end{array}$ \\
\hline TI-201 & $73.1 \mathrm{~h}$ & $\begin{array}{l}\text { Clinical cardiology (C), heart imaging (C), less desirable nuclear characteristics than } \\
\text { Tc-99m for planar and SPECT imaging (C), myocardial perfusion, cellular dosimetry. }\end{array}$ \\
\hline $\mathrm{Tm}-170$ & $129 d$ & Portable blood irradiations for leukemia, lymphoma treatment, power source. \\
\hline Tm-171 & $1.9 y$ & Medical. \\
\hline W-188 & $69.4 d$ & Cancer treatment, monoclonal antibodies, parent for Re-188 generator. \\
\hline Xe-127 & $36.4 d$ & $\begin{array}{l}\text { Neuroimaging for brain disorders, research for variety of neuropsychiatric disorders, } \\
\text { especially schizophrenia and dementia, higher resolution SPECT studies with lower } \\
\text { patient dose, lung imaging (some experts believe it is superior to Xe-133 in inhalation } \\
\text { lung studies). }\end{array}$ \\
\hline Xe-133 & $5.25 d$ & $\begin{array}{l}\text { Lung imaging }(C) \text {, regional cerebral blood flow (C), liver imaging (gas inhalation) (C), } \\
\text { SPECT imaging of brain, lung scanning, lesion detection. }\end{array}$ \\
\hline $\mathrm{Y}-88$ & $107 d$ & Substituted for Y-90 in development of cancer tumor therapy. \\
\hline Y-90 & $64 \mathrm{~h}$ & $\begin{array}{l}\text { Internal radiation therapy of liver cancer }(C) \text {, monoclonal antibodies }(C) \text {, Hodgkins } \\
\text { disease, and hepatoma }(C) \text {, cellular dosimetry, treating rheumatoid arthritis, treating } \\
\text { breast cancer, treatment of gastrointestinal adenocarcinomas }(A) \text {. }\end{array}$ \\
\hline Y-91 & $58.5 d$ & Cancer treatment (RIT), cellular dosimetry. \\
\hline Yb-169 & $32 d$ & Gastrointestinal tract diagnosis. \\
\hline Zn-62 & $9.22 \mathrm{~h}$ & $\begin{array}{l}\text { Parent of Cu-62, a positron-emitter, used for the study of cerebral and myocardial blood } \\
\text { flow. }\end{array}$ \\
\hline Zn-65 & 244d & Medical. \\
\hline Zr-95 & $64.0 \mathrm{~d}$ & Medical. \\
\hline
\end{tabular}

NOTES:

A = June 1996 SNM Abstracts

$\mathrm{B}=$ Holmes 91

C $=$ Herac 89

$\mathrm{D}=$ Fairchild 87

$\mathrm{E}=$ Everyone's Guide to Cancer Therapy (Dollinger, Rosenbaum, Cable), 1991

$\mathrm{F}=\mathrm{SNM}$ (Society of Nuclear Medicine) 